\title{
Should local research ethics committees monitor research they have approved?
}

Emma Pickworth University of Manchester

\begin{abstract}
The function of local research ethics committees is to consider the ethics of research proposals using human participants. After approval has been given, there is no comprehensive system in place to monitor research and ensure that recommendations are carried out. Some suggest that research ethics committees are ideally placed to fulfil this function by carrying out random monitoring of research they have reviewed. The health service guideline creating local research ethics committees is under review. ${ }^{1}$ This paper suggests that increasing the monitoring role of ethics committees in the present climate would be inappropriate. This is due to the large workload of the committees, their voluntary nature and the change a monitoring role might cause to the relationship between researcher and ethics committee, which might herald an increasing recourse to judicial review. A radical overhaul of the system would be necessary in order for ethics committees adequately to fulfil a monitoring function.

(Fournal of Medical Ethics 2000;26:330-333)
\end{abstract}

Keywords: Research ethics committees; audit; monitoring; misconduct

\section{Introduction}

In 1991 a health service guideline was issued stating that "every health district should have a local research ethics committee to advise NHS [National Health Service] bodies on the ethical acceptability of research proposals involving human subjects". "This paper considers whether their role is indeed limited to a consideration of "research proposals involving human subjects", or is gradually evolving to incorporate a duty to review and monitor the research after the initial approval.

Local research ethics committee (LREC) members come from a variety of backgrounds and rarely receive recompense for the considerable time they spend reading and deliberating over research proposals. The LREC workload is extensive. Funding is often inadequate and many committees have resorted to charging for review of commercially sponsored proposals in order to raise funds for training and administration. ${ }^{3}$ This contrasts sharply with international equivalents where the administrative and funding systems are often more conducive to a monitoring role. Nevertheless, it is conceivable that pressure will be brought to bear on LRECs to increase their monitoring function.

Though there are a variety of monitoring systems within the UK, we currently lack a comprehensive system to which all health care research is subjected. Thus, in isolation, the Medical Controls Agency monitors serious adverse events in clinical trials, the Research and Development Directorate of the NHS Executive monitor management and finance, and research ethics committees monitor progress of the research they review.

There are three loosely defined categories which LRECs could adopt to try and protect research participants when monitoring research after approving a protocol:

- detecting fraudulent research;

- collecting progress reports and reviewing changes to protocols, and

- proactive monitoring through questionnaire and/or visitation of research site.

In relation to the first category, detecting fraudulent research involves an expensive policing role, largely inappropriate for LRECs which are concerned with facilitating scientifically valid research and protecting the dignity and welfare of research participants. The current monitoring processes of LRECs are largely confined to the second category, collecting progress reports and reviewing changes to protocols, with the exception of a small number of committees which have extended their monitoring activities to the third category. Local research ethics committees have access to the original research protocol and are arguably in an ideal position to increase their monitoring function. However, though there is evident value in proactive LREC monitoring, a greater commitment to adequate resourcing and further professional guidance is needed before it can be more widely adopted.

\section{Limited role of ethics committees in detecting fraudulent research}

Fraudulent research has attracted significant media attention, not least because it has proved remarkably difficult to detect. Career advancement is partially dependent on research publications, and the funding obtained as a result makes fabrication a tempting shortcut to some. In 1996 a researcher forged two letters purporting to come from the Salford research ethics committee giving approval for the research to go ahead ${ }^{4}$; and in November 1997 a senior physician and a former registrar of the Royal College of Physicians of Edinburgh were struck off for conducting a fifteen-month fraudulent drug trial. The trial attracted significant media interest 
because of the potentially fatal consequences of doctors acting on false information. ${ }^{5}$ Again, in 1998 a paper was withdrawn from the British Medical fournal because of a researcher's misconduct and lies concerning his qualifications. ${ }^{6}$

This area is currently overseen by the Medical Research Council ${ }^{7}$ together with the Committee on Publication Ethics, ${ }^{8}$ aided by the guidance of the Royal College of Physicians' report, $1991 .{ }^{9}$ In view of the fact that fraud often involves omitting the review process altogether, the detecting role of LRECs is likely to remain insubstantial. However, in the limited circumstances where the fraudulent behaviour involves non-adherence to the approved protocol, it is possible that widening the role of LRECs to include random monitoring of research they have approved might deter, detect and reduce cases of research misconduct.

\section{Collecting progress reports and reviewing changes to protocols}

Professional guidelines place duties on researchers to report progress and changes of protocol to the relevant ethics committee(s). ${ }^{10}$ Adverse reactions in clinical research protocols are reported to the Medical Controls Agency (MCA). Health service guideline (91)5 at paragraph 2.14 also states that: "Reports to the [local research ethics] committee should also be required once the research is underway if there are any unusual or unexpected results which raise questions about the safety of the research". ${ }^{1}$ However, there is no agreed operative procedure for dealing with such reports which, in some circumstances, are merely filed away. In the case of academic research protocols, in which adverse reactions are not reported to the MCA, a standardised procedure for dealing with reports would be especially useful.

The Health Service Guideline (91) 5 introducing the LREC system states that: "If it comes to the attention of a committee ... that its recommendations have been ignored, then the LREC should bring the matter to the attention of its appointing authority, ..." but this places no duty on the committee actively to audit investigators. ${ }^{1}$ The 1996 Royal College of Physicians' guidance, published three months after the International Conference on Harmonisation Good Clinical Practice guidelines, adds somewhat to these duties. The report recognises the impracticalities of RECs actively monitoring research, but encourages it as an occasional endeavour. ${ }^{11}$ However, this guidance has only limited force for LRECs which are bound by the health service guideline.

\section{Inconsistent protection for research participants}

In commercially sponsored trials, a monitor is usually appointed to guard against and react to a range of occurrences, as is verified in the International Conference on Harmonisation Good Clinical Practice guidelines. ${ }^{12}$ In investigations involving drugs or novel equipment and trials sponsored by industry, reporting adverse events to the sponsor is mandatory under the Good Clinical Practice Guidelines issued by the Association of the British Pharmaceutical Industry (1993). ${ }^{13}$ In other types of research, participants are reliant on the investigators conducting the research according to the approved protocol and faithfully reporting any divergence or alteration to the LREC. In the light of those cases of fraud outlined above, this is an inadequate means of protecting research participants.

\section{Pressure for LRECs to increase their monitoring role}

Not only is the current monitoring system insufficient, but calls are being made to increase and harmonise monitoring procedures (particularly in relation to clinical trials) both across Europe ${ }^{14}$ and internationally. In the USA, the rules governing institutional review boards (which are roughly the equivalent of our research ethics committees) have recently come under a great deal of scrutiny. Amongst the recommendations in a 1998 Inspector General's report ${ }^{15}$ was the need for a universal system of monitoring trials. The report suggested that institutional review boards could take a more active role in this.

In Australia the law was amended in 1992 to require institutional ethics committees to monitor research projects after they received ethical approval. ${ }^{16}$ As in the UK, emphasis is placed on reporting by the investigator. Hence the investigator is required to make at least an annual report and to notify the institutional ethics committee of adverse effects, changes in protocol and unforeseen events. The institutional ethics committee is given the possible sanctions of withdrawing ethical approval or informing the governing body of the institution who can then take disciplinary action against the investigators. There is pressure ${ }^{17}$ for a system enabling audit of a random selection of research project records; the appointment of a member of the institutional ethics committee to act as a monitor for specific projects; the establishment of a subcommittee to review progress reports, and the establishment of a complaint-handling mechanism within the institutional ethics committee for research participants to contact. Further suggestions include making approval subject to the signed agreement of investigators to comply with the regulations, requiring researchers to report to the institutional ethics committee every six months and enforcing the publication of results.

The international impetus for increased monitoring of trials by ethics committees may have an effect on LRECs. However, though there are similarities in the roles of the various committees, it should be noted that the US and Australian committees are usually attached to an institution, unlike the LREC which is independent. They serve that institution, protecting it from legal liability. It is therefore conceivable that their role should not cease once the protocol has been approved. In short, for the LREC to perform the duties starting to be requested of their international equivalents, a degree of change 
is needed. This is not only in terms of funding, but also in terms of the very nature of the independent, voluntary basis of the LREC.

The Department of Health guidance to LRECs supports the current, limited monitoring functions of LRECs. As health service guideline (91) 5 is currently under review, it remains to be seen whether the Department of Health will increase the LREC monitoring role, allocate it to another branch of the system such as the Research and Development Directorate of the NHS Executive, or allow the current divergences between the monitoring in clinical and non-clinical research settings to remain.

\section{Proactive monitoring through questionnaire and/or visitation of research site}

The case for extending the LREC monitoring role is based on the fact that LRECs' approval of the protocol and receipt of progress reports makes them obvious candidates to fill an evident gap in a system designed to protect research participants from unethical research. Unfortunately, though sanctions are issued by the National Health Service (NHS) against any of its employees who initiate research on human subjects without REC approval, the LREC is currently in an inadequate position to ensure that all researchers adhere to the agreed protocol.

Nevertheless, random monitoring by LRECs has proved successful, where adequate time and funding have been obtained. For example, the Tayside Committee on Medical Research Ethics ${ }^{18}$ published results of a monitoring exercise in 1997. The process sought to measure adherence to the approved protocol in 39 projects, particularly in terms of recruitment and specific requirements of the ethics committee (such as notification of changes and adverse reactions). This monitoring exercise produced startling results. In over a quarter of the projects there were divergences from the protocol in relation to the consent process. Though adverse events were reported, projects which were abandoned or late to start were vastly underreported (of the 39 projects, nine were abandoned and only one of these was reported to the committee). Neither was the ethics committee always informed of relevant changes to the protocol. Tayside continues randomly to monitor research projects by means of questionnaire and visitation.

Berry ${ }^{19}$ the chairman of Mid Downs East LREC, conducted a slightly different monitoring exercise. Berry selected a researcher sponsored commercially (so that some monitoring was already in place), and wrote to participants in order to check the standards by means of a questionnaire. Berry counsels that the questionnaire be kept short and simple and that the research participants agree to their name and addresses being passed to the committee before audit takes place, and that they are made aware that they are under no obligation to answer the questions. The questionnaire focused on the information received, the consent given, inducements received and instructions in the event of adverse effects. In this instance, the results showed an acceptable level of compliance.

\section{Problems with LREC monitoring}

Though there have been isolated examples of LREC monitoring, there are prohibitive time and resource implications to it becoming more widespread. Berry suggests that one option is to build the costs into fees charged to commercially sponsored research where this is already the practice of the LREC.

Another problem relates to multicentre research ethics committee (MREC) approved research. Multicentre research ethics committees review research that is carried out in five or more LREC geographic localities. The protocol is then sent to each LREC in order that the committee can consider local issues. One MREC decision is good across the whole of the UK. Therefore, though progress reports, changes to protocol and adverse events should be reported to both the MREC and LREC, proactive monitoring could be an extremely costly and impractical process if performed by the MREC. For this reason it is expected that any proactive REC monitoring will be a local rather than a multicentre activity.

Local research ethics committees might also be opposed to monitoring research on the basis that it may alter their relationship with researchers. Their current role, guiding researchers as to the ethicality of the protocol, would be extended into what might be perceived as a policing role. Disgruntled researchers may be increasingly likely to opt for legal action (in particular, judicial review) where they believe the process to have been unfair. This is all the more likely if LRECs charge a fee in order to fund their extra monitoring role, as this potentially creates a contract between the researcher and the health authority. ${ }^{20}$ Consequently, unless the reform of the health service guideline radically alters the current situation, LREC audit will at best be an infrequent event.

\section{Conclusion}

The guidance that LRECs should perform occasional monitoring leaves in the hands of the LREC the frequency and detail of the audit. However, the guidance expresses it as a desirable function and it is clear that some sort of monitoring system, that is not purely reliant on the honesty of investigators, would be desirable in protecting research participants. This is especially so in non-clinical research where monitoring requirements are often far less stringent than is the case in pharmaceutically sponsored clinical research. Health service guideline (91) 5 is currently under review within the Department of Health, and the monitoring duties of RECs is one issue under consideration. If it is decided that LRECs should increase their monitoring role, then there must be some means of financing the admin- 
istration and possibly even the reasonable expenses of LREC members. The appropriate role of MRECs in relation to audit, will also need to be clarified. To protect members from potential judicial review, the process and procedures should be carefully set out, covering the issues of how frequently to audit, what types of research to audit, and the appropriate method. If a questionnaire is used in the monitoring process, consensus on the appropriate format is needed. It must be ascertained whether the questionnaire will consider participants' perceptions or the rigour with which the REC recommendations are carried out.

It is suggested that the independent nature of ethics committees in the UK, coupled with their limited time, voluntary nature and large workload, make extensions of their current monitoring duties inappropriate. The current drive for clinical governance makes monitoring of research highly appropriate but, contrary to some international experience, it may be a role more suited to the Research and Development Directorate of the NHS Executive than research ethics committees. The Culyer report, Supporting Research and Development in the NHS, ${ }^{21}$ was critical of the complex funding arrangements for $\mathrm{R} \& \mathrm{D}$ and recommended a single explicit funding mechanism for both direct and indirect costs of $\mathrm{R} \& \mathrm{D}$ projects and programmes, including the costs of maintaining facilities enabling $\mathrm{R} \& \mathrm{D}$ programmes to take place. Ensuring the safety of research participants is integral in the maintenance of an ethical R\&D programme and could therefore legitimately be placed within the ambits of $R \& D$.

\section{Acknowledgement}

The author appreciates the support of the Liverpool, Manchester, Preston Ethics Training (LiMPET) Project, a collaborative venture between the Universities of Central Lancashire, Manchester, and Liverpool, funded by the NHS Executive North West.

Emma Pickworth, LLB, Mfur, was a Research Fellow at the Centre for Professional Ethics, University of Central Lancashire, Preston when she wrote this paper. She is currently a Researcher at the Institute of Medicine, Law and Bioethics, University of Manchester.

\section{References and notes}

1 Department of Health. Local research ethics committees. London: Department of Health, undated but published in August 1991 as health service guideline HSG(91)5. Neuberger J. Ethics and health care: the role of research ethics committees in the United Kinghealth care: the role of research ethics committees in the United King-

2 Nom [research report 13]. London: King's Fund Institute, 1992. 2 Nicholson R. What do they get up to? A study of L

3 Dr Fyoti Kamar Agarwala v The General Medical Council (1996) Lawtel doc no C0005006 (unreported elsewhere)

4 Slapper G. Doctored research. The Times 1997 Nov 4: 41.

5 Dyer C. Doctor admits research fraud. British Medical fournal 1998;316:645.

6 Smith R. The need for a national body for research misconduct. British Medical fournal 1998;316:1686-7.

7 Evans I. Conduct unbecoming - the MRC's approach. British Medical fournal 1998;316:1730.

8 Smith R. Misconduct in research: editors respond: the Smith R. Misconduct in research: editors respond: the
committee on publication ethics (COPE) is formed. British committee on publication ethics

9 Royal College of Physicians. Fraud and misconduct in medical research. London: Royal College of Practitioners, 1991

10 Department of Health. Standards for LRECs - a framework for ethical review. London: Department of Health, 1994: module 17.

11 Royal College of Physicians. Guidelines on the practice of ethics committees in medical research involving human subjects. London: Royal College of Physicians, 1996: paras 2.12 and 2.14.

12 International Conference on Harmonisation of Technical Requirements for Registration of Pharmaceuticals for Human Requirements for Registration of Pharmaceuticals for Human
Use. ICH harmonised tripartite guideline: guideline for good clinical Use. ICH harmonised tripartite guideline:

13 Association of the British Pharmaceutical Industry. Good clinical practice guidelines. London: ABPI, 1993.

14 European Commission. Proposal for a European parliament and council directive on the approximation of provisions laid down by law, regulation or administrative action relating to the implementation of good clinical practice in the conduct of clinical trials on medicinal products for human use. Official fournal of the European Communities 1997;C306:10: article 12.

15 Department of Health and Human Service's Inspector General's Report. Institutional review boards: a time for reform. Washington, DC: Office of Inspector General, Department of Health and Human Services, 1998 Jun 11.

16 National Health and Medical Research Council of Australia. Statement on human experimentation. Australia: National Health Statement on human experimentation. Australia: National Health and $M$

17 Linden-Laufer S. Monitoring approved research protocols - a question of balance. Medicine and Law 1997;16:655-77.

18 Smith T, Moore EJH, Tunstall-Pedoe H. Review by a local medical research ethics committee of the conduct of approved research projects, by examination of patients' case notes, consent forms, and research records and by interview. British Medical fournal 1997;314:1588.

19 Berry J. LRECs can audit ethical standards in research. Fournal of Medical Ethics 1997;23:379-81.

20 Pickworth E, Brazier M. Fees and research ethics committees. Bulletin of Medical Ethics 1999;151:18.

21 NHS Research and Development Task Force. Supporting research and development in the NHS. London: Department of Health, 1994. 